\title{
Image-guided radiation therapy using surgical clips for localization of colonic metastasis from thyroid cancer
}

\author{
Alvin Szeto ${ }^{1}$, Lee Chin ${ }^{1}$, Patrick Whelan², Jennifer Wilson ${ }^{3}$ and Justin Lee ${ }^{1 *}$
}

\begin{abstract}
A 67-year old man with a history of papillary thyroid cancer (PTC) presented with metastatic disease to the left colon in the form of a $6.1 \times 1.0 \mathrm{~cm}$ bleeding, ulcerated mass. Radiopaque surgical clips were used as fiducial markers to localize the gross tumor volume (GTV) as well as the corresponding clinical target volume (CTV) and planning target volume (PTV). Daily cone beam computed tomography (CBCT) image guidance was utilized to verify the tumor position. Inter- and intrafraction movement of the tumor mass was assessed. Gastrointestinal bleeding was controlled using palliative image-guided radiation therapy (IGRT).
\end{abstract}

Keywords: Fiducial markers, Image-guided radiation therapy, Surgical clips, Thyroid cancer, Colonic metastasis, Lower gastrointestinal bleed

\section{Background}

Papillary thyroid cancer (PTC) is the most common form of well differentiated thyroid cancer and is usually associated with good prognosis [1]. Recurrence rates are reported between $10-33 \%$ depending on stage and histological subtype [2]. Common sites for distant metastases of PTC include mediastinal lymph nodes, lung and bone [3]. Here, we discuss a patient with recurrent PTC with distant metastases to mediastinal lymph nodes, lung, spine, as well as colon. Gastrointestinal metastases of thyroid cancer account for $0.5-1 \%$ metastatic sites [3]. To date, there are only a small number of patient reports documenting metastatic disease from the thyroid to the colon [4-6].

Radiation treatment of tumors of the colon is not typically performed as it presents a challenge to visualize and consistently target mobile abdominal organs. Treatment planning without adequate visualization of the target area can result in wide treatment margins and excessive toxicity. Movement of the intra-abdominal organs can result in underdosing of the target or overdosing of the surrounding organs. Furthermore, conventional CBCT

\footnotetext{
* Correspondence: justin.lee@sunnybrook.ca

'Department of Radiation Oncology, Sunnybrook Odette Cancer Centre, University of Toronto, 2075 Bayview Avenue, Toronto, ON M4N 3M5, Canada Full list of author information is available at the end of the article
}

techniques rely on bony matching, which does not necessarily correlate with internal organ motion. Use of fiducial markers, such as surgical clips, can narrow the treatment volume and improve radiation targeting. Previous studies demonstrate that use of surgical clips for targeting prostate cancer, results in a reduction in total setup error, smaller planning target volume (PTV) margin and rectal sparing [7]. Despite these potential benefits, fiducial markers may also have a negative impact on clinical outcomes if the PTV margin is significantly reduced without careful consideration of intrafraction organ motion. [8].

Here, we present a unique case of multi-site metastasis of papillary thyroid carcinoma including an ulcerated, bleeding colonic mass. Furthermore, we describe our experience with image-guided radiation therapy (IGRT) for palliative treatment to control bleeding of metastatic disease to the colon.

\section{Case presentation}

A 67-year old man presented with an ulcerated, bleeding mass in the colon, shown by biopsy to be metastatic PTC in May 2014.

He initially presented with a locally advanced thyroid tumour in 2005 treated with total thyroidectomy and bilateral neck dissection. Post-surgical pathology revealed the primary lesion to be classic papillary thyroid 
carcinoma with areas of follicular and focal oncocytic variants. There was evidence of capsular invasion, extrathyroidal extension involving muscle, adherence to the trachea and involved neck nodes; tumour stage was pT4N2M0. The patient underwent adjuvant radioactive iodine, and external beam radiation to a dose of 60Gy over 30 fractions to the thyroid bed and neck.

In June 2007, the patient developed locoregional recurrence which was treated with left neck dissection and radioactive iodine of 150 millicuries. In December 2009, chest imaging revealed pulmonary and mediastinal metastases which were treated with a third course of radioactive iodine of 157 millicuries. In January 2013, the patient presented with spinal metastases involving the T9, T11, and L2 vertebrae encroaching on the spinal canal. This was treated with radiation dose of 20Gy in 5 fractions to control neuropathy and bony pain.

In June 2014, the patient presented with abdominal fullness and lower gastrointestinal bleeding. Abdominal and pelvic CT with contrast showed metastatic deposits throughout the abdomen and pelvis, with lymphadenopathy involving the gastrohepatic ligament, celiac axis, aortocaval and left external iliac regions. The patient was found to have a hemoglobin ( $\mathrm{HgB}$ ) level of $65 \mathrm{mg} / \mathrm{L}$ and was transfused a total of 4 units of packed red blood cells.

Colonoscopy was performed; an actively bleeding $6.1 \times$ $1.0 \mathrm{~cm}$ ulcerated lesion was dominant in the left colon. Biopsy of the colon mass was performed and the immunohistochemical profile was positive for TTF-1 and CK7, negative for CDX2, CK20 and HBME1. Immunohistochemistry and morphology were consistent with metastatic papillary thyroid cancer. Further surgical management of the mass was not deemed appropriate given the patient's extent of disease and comorbidities. Additional radioactive iodine treatment was not recommended after considering the three previous doses and the low likelihood of targeting the colonic lesion. Therefore, the patient was planned for radiotherapy with the intent to stop bleeding from the colonic mass. A palliative dose of 20 Gy in 5 fractions was chosen to try to alleviate symptoms while limiting toxicity to the adjacent, unaffected organs at risk.

\section{Colon tumor localization}

The initial colonoscopy described the location of the mass to be distal to the splenic flexure. However, the mass could not be resolved on diagnostic CT with contrast or initial CT simulation. Due to this uncertainty, the initial radiation treatment CTV and PTV encompassed the entire descending colon (Figure 1). It was felt that this large irradiated volume might cause excessive acute gastrointestinal side effects. Therefore, to better localize the colonic metastasis, three metallic surgical clips were inserted during repeat colonoscopy: proximal, distal and one within the ulcerated mass. On re-simulation, the surgical clips were found to be in the left lower quadrant just proximal to the sigmoid colon. The GTV was contoured to encompass the three clips and the segment of colon defined by the ends of the clips. Due to uncertainties regarding the extent of disease and intrafraction organ motion, a volumetric CTV expansion of $1 \mathrm{~cm}$ was applied followed by an additional PTV margin of $1 \mathrm{~cm}$. Localization with the clips reduced the PTV from $393.8 \mathrm{~cm}^{3}$ to $251.5 \mathrm{~cm}^{3}$

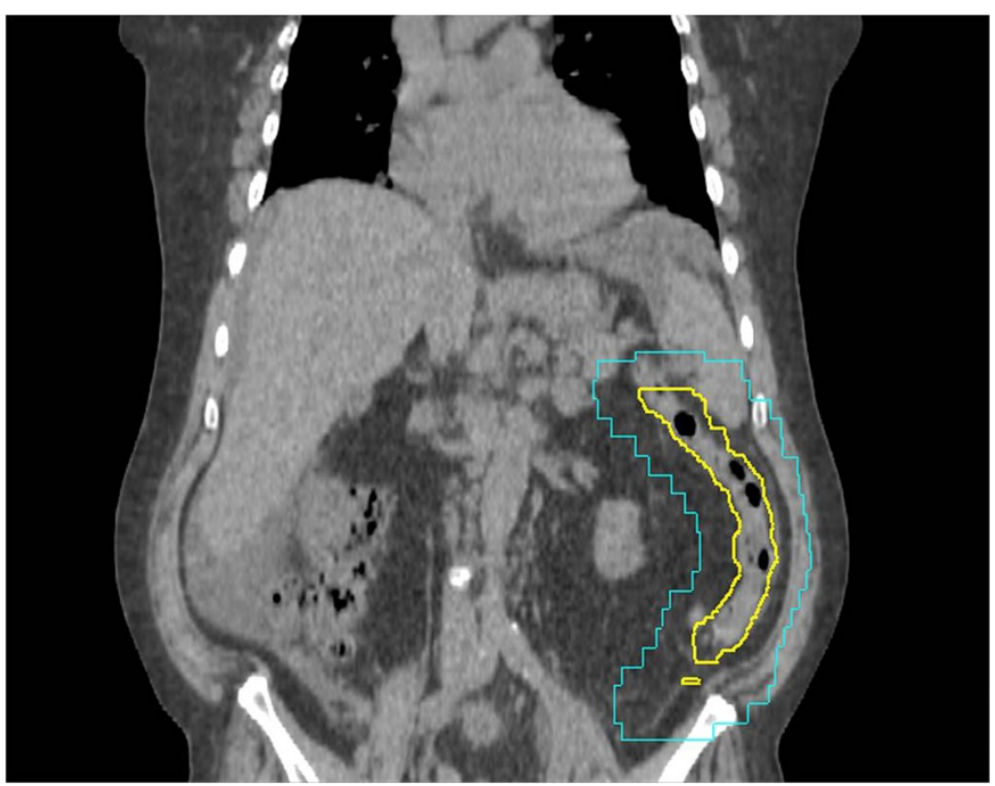

Figure 1 Computed tomography of palliative radiotherapy plan without clips. Representative coronal CT image of the CTV (yellow) and radiation field edges (blue) without fiducial markers present. No GTV was visible therefore the CTV contour included the entire descending colon. 
while shifting the geometric center of the PTV by $13.7 \mathrm{~cm}$ (Figure 2).

\section{Clinical treatment technique: image guided radiotherapy with colon fiducial markers}

Simulation and treatment were performed with the patient in the supine position, without contrast, using a knee support for comfort. Bladder filling was not specified for the simulation or treatment.

On treatment, daily $\mathrm{CBCT}$ verification was performed using image fusion from the Pinnacle 9.2 Treatment Planning System (TPS; Philips Healthcare, Andover, MA) to ensure the PTV encompassed the three clips and CTV contour. Bony registration was first confirmed on preliminary $\mathrm{CBCT}$ and then, if required, any additional shifts were applied to ensure that all three clips were encompassed by the PTV volume. The three clips were well visualized by radiation therapy staff and centered within the PTV volume on each of the five treatment days.

\section{Analysis of inter- and intrafractional organ movement} A review of each daily CBCT image and treatment shifts was performed to determine tumour motion using the surgical clips as a surrogate to define the GTV. The clips were contoured in the initial CT simulation scan and all subsequent $\mathrm{CBCT}$ images.

Clip displacement (i.e. inter- and intrafractional movement of the mass) was assessed using Image Fusion from the Pinnacle 9.2 TPS. Cone beam CT images from each treatment day were fused with the initial planning CT for bony registration. Displacement was calculated by comparing the coordinates of the geometric centers of the contoured clip volume (i.e. the GTV) of the initial planning CT versus daily CBCTs. The average interfraction movement of the clips was $0.69 \mathrm{~cm}$ (ranging 0.51-0.98 cm) from initial planning (Table 1).

On days 4 and 5 of treatment, the patient required additional repositioning/shifting to center the PTV onto the target. Intrafractional movement (i.e. the difference between daily pre- and post-shift movement) of the patient produced a $0.24 \mathrm{~cm}$ and $0.41 \mathrm{~cm}$ displacement of the target on days 4 and 5 , respectively (Table 2). The clips had the greatest mean magnitude of displacement in the leftright dimension $(0.83 \mathrm{~cm})$, followed by superior-inferior $(0.51 \mathrm{~cm})$ and antero-posterior $(0.44 \mathrm{~cm})$ dimensions (data not shown). As inter- and intra-fractional displacement is not yet well understood for tumours located within the colon, PTV margins were maintained at $1 \mathrm{~cm}$ throughout the treatment course to ensure adequate coverage.

\section{Clinical follow-up}

The patient tolerated the palliative course of radiation treatment well with no gastrointestinal or genitourinary toxicity observed. Lower gastrointestinal bleeding stopped within 4 days of completing radiation therapy and posttreatment $\mathrm{HgB}$ level was $95 \mathrm{mg} / \mathrm{L}$; no additional transfusions were required. At six-week follow-up, the patient did not have any recurrent lower GI bleeding and $\mathrm{HgB}$

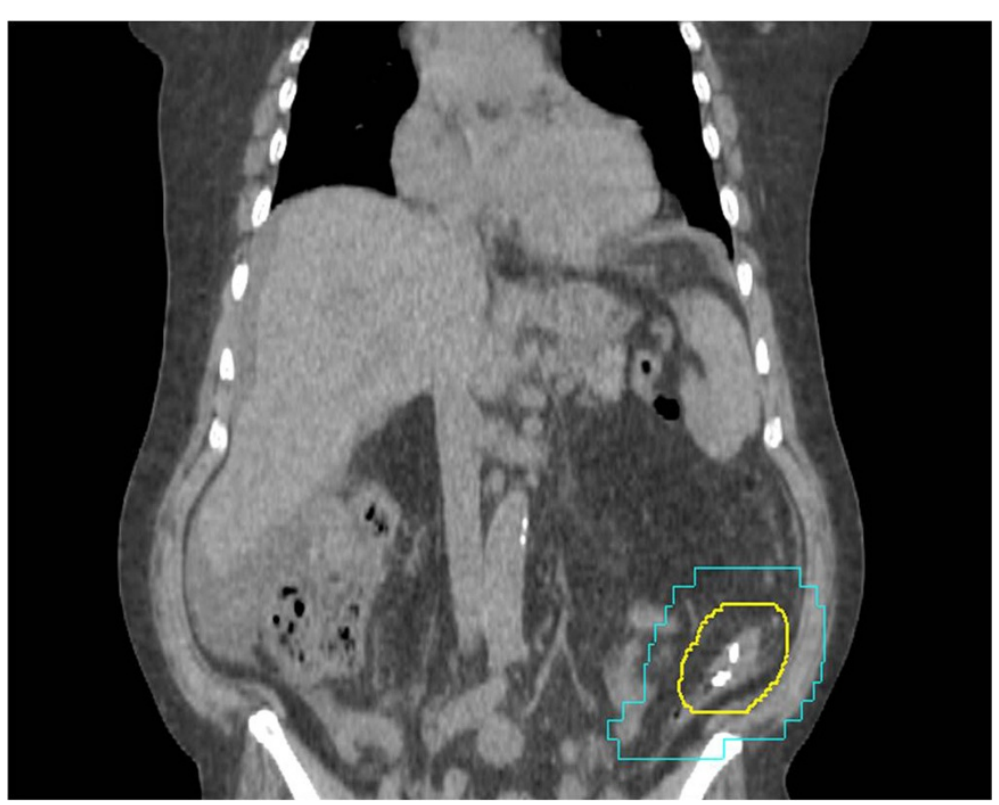

Figure 2 Computed tomography of palliative radiotherapy plan with clips placed. Representative coronal CT image of the CTV (yellow) and radiation field edges (blue). Three clips were placed within the tumour and at the distal and proximal extent. The PTV volume was decreased from $393.8 \mathrm{~cm}^{3}$ to $251.5 \mathrm{~cm}^{3}$. The V15 for small bowel loops was reduced from $328.0 \mathrm{~cm}^{3}$ to $176.0 \mathrm{~cm}^{3}$. 
Table 1 Interfractional displacement of surgical clips on treatment day from planning

\begin{tabular}{ll}
\hline Treatment day & Interfractional movement $\mathbf{( c m )}$ \\
\hline 0 (Planning) & -- \\
1 & 0.98 \\
2 & 0.51 \\
3 & 0.55 \\
4 & 0.61 \\
5 & 0.64 \\
\hline
\end{tabular}

CBCT images were taken at planning and before each day of treatment. Images were fused with the planning $\mathrm{CT}$ image after bony registration. Displacement of the clips between the each day and planning were calculated. Pre- and post-shift displacements are measured from the central coordinates of the GTV.

had increased to $103 \mathrm{mg} / \mathrm{L}$. At a later date, the patient was admitted to hospital with thromboembolic stroke and died from complications.

\section{Discussion}

Given the complicated and unfortunate course of disease for this patient, palliative management was recommended to control lower GI bleeding, avoid further transfusions, and improve quality of life. Rare abdominal and pelvic metastasis of papillary thyroid cancer presented a challenge for management, and only a handful of reports have been published [4-6]. One report documented a 19-year old girl with a rare cribriform-morular variant of PTC, associated with familial adenomatous polyposis syndrome [4]. Another report described Hurthle cell thyroid carcinoma metastatic to the sigmoid colon with radioactive iodine resistance [6]. However, these studies were primarily treated with surgery and did not utilize external beam radiation therapy for the abdominal and pelvic metastases. As noted, surgery for this patient was contraindicated due to the extent of metastatic disease and comorbidities.

Abdominal organ and tumor movements are influenced by respiration and bladder filling status, leading to irregular changes in conformity [9]. Abbas et al. reviewed multiple motion management strategies to control abdominal organ movement for radiation therapy, including motion control/suppression methods and respiratory gating during treatment; however, movement of the intestines may present additional challenges due to the relatively unfixed

Table 2 Intrafractional movement of the GTV on Day 4 \& 5

\begin{tabular}{llll}
\hline $\begin{array}{l}\text { Treatment } \\
\text { day }\end{array}$ & $\begin{array}{l}\text { Pre-shift } \\
\text { displacement } \\
\text { (cm) }\end{array}$ & $\begin{array}{l}\text { Post-shift } \\
\text { displacement } \\
\text { (cm) }\end{array}$ & $\begin{array}{l}\text { Change in } \\
\text { displacement } \\
\text { (cm) }\end{array}$ \\
\hline 4 & 0.61 & 0.44 & 0.21 \\
5 & 0.64 & 0.35 & 0.41 \\
\hline
\end{tabular}

The patient was repositioned on days 4 and 5 after initial CBCT to improve GTV alignment and conform to PTV margins. The change in magnitude of intrafractional displacement is shown. nature of the organs compared to other abdominal organs [9].

Irradiation of the abdomen and pelvis may cause acute toxicities including nausea, vomiting and diarrhea. For palliative dose regimens such as 20 Gy in 5 fractions the reported rates of nausea and/or vomiting range between 30-50\% [10-12]. Biological models and clinical studies suggest that the volume of small bowel loops receiving 15 Gy or more (V15) may be an important factor. A threshold of V15 $>120 \mathrm{~cm}^{3}$ is associated with $>10 \%$ risk of grade 3 or higher acute toxicity [13-15]. We retrospectively contoured the loops of small bowel and evaluated the V15 for the radiotherapy plans with and without fiducial markers. The preliminary radiation plan resulted in a V15 of $328 \mathrm{~cm}^{3}$ which would have exposed the patient to a high risk of severe acute small bowel toxicity[13]. The plan based on surgical clips for target delineation yielded close to $50 \%$ reduction in the small bowel dose, with a V15 of $176 \mathrm{~cm}^{3}$, suggesting a potential decrease in the risk of severe toxicity.

Inter- and intrafractional tumor movement have been assessed with regards to lung, pancreatic, prostatic, vaginal and hepatic cancers, but not lesions in the colon [16-20]. Recent case studies have also employed the use of surgical clips as fiducial markers in radiosensitive intra-abdominal lymphomas in the duodenum and colon but did not report on tumour movement and adequacy of PTV margins [7,9]. Other studies have assessed the use of fiducial markers and daily $\mathrm{CBCT}$ registration in bladder, pancreas and prostate, which have found significant discrepancies between interfractional fiducial marker location and bony anatomy [21-23]. Despite the improved tumor visualization with fiducial markers, proper organ motion data must be assessed to accurately delineate the target margins to avoid inappropriate dosing [8]. In this case, CBCT was used to first ensure bony matching and then to confirm tumour coverage. The clip displacements after bony registration ranged between $0.21-0.98 \mathrm{~cm}$, therefore additional shifts were not required and the clinical impact was limited. In future studies, clip displacement might be used to evaluate systematic errors and reduce PTV margins.

\section{Conclusion}

Here we present a rare case of papillary thyroid cancer metastasis to the colon treated with a palliative dose of IGRT to control lower GI bleeding. Surgical clips were used as fiducial markers with a volumetric expansion of $1 \mathrm{~cm}$ for the CTV and $1 \mathrm{~cm}$ for the PTV. The main benefit of the fiducial markers in this case was accurate delineation of the GTV which allowed for smaller target volumes. Using daily $\mathrm{CBCT}$, the interfraction and intrafraction clip movements were measured to be less than $1 \mathrm{~cm}$ which was within the PTV margin chosen for this case. 
This study demonstrates the clinical efficacy of palliative IGRT for a colon tumor using surgical clips as fiducial markers to enhance target localization and verify daily treatment position. The radiopaque surgical clips provide a reliable marker for visual verification of a mobile and otherwise imperceptible lesion.

\section{Consent}

Informed consent was obtained from the patient and next of kin for publication of this case report and any accompanying images.

\section{Abbreviations}

PTC: Papillary thyroid cancer; IGRT: Image-guided radiation therapy; GTV: Gross tumor volume; PTV: Planning target volume; CBCT: Cone beam computed tomography; GERD: Gastroesophageal reflux disease; TPS: Treatment planning system.

\section{Competing interests}

The authors declare that they have no competing interests.

\section{Authors' contributions}

AS researched and drafted the manuscript. AS and LC collected the data and performed the analysis. $J \mathrm{~L}$ was the primary radiation oncologist involved in this case. $J$ and LC substantially contributed to drafting and revision of the manuscript. PW was the primary surgeon involved in the management of this case. JW was the primary care physician managing the patient's care. All authors read and approved the final manuscript.

\section{Author details}

'Department of Radiation Oncology, Sunnybrook Odette Cancer Centre, University of Toronto, 2075 Bayview Avenue, Toronto, ON M4N 3M5, Canada. ${ }^{2}$ Department of Surgery, Markham Stouffville Hospital, Markham, Ontario, Canada. ${ }^{3}$ Department of Family and Community Medicine, Markham Stouffville Hospital, Markham, Ontario, Canada.

Received: 31 October 2014 Accepted: 12 December 2014

Published online: 24 December 2014

\section{References}

1. Chrisoulidou A, Boudina M, Tzemailas A, Doumala E, lliadou PK, Patakiouta F, Pazaitou-Panayiotou K: Histological subtype is the most important determinant of survival in metastatic papillary thyroid cancer. Thyroid Res 2011, 4:12.

2. Shaha AR, Ferlito A, Rinaldo A: Distant metastases from thyroid and parathyroid cancer. ORL J Otorhinolaryngol Relat Spec 2001, 63:243-249.

3. Klubo-Gwiezdzinska J, Morowitz D, Van Nostrand D, Burman KD, Vasko V, Soberman M, Wartofsky L: Metastases of well-differentiated thyroid cancer to the gastrointestinal system. Thyroid 2010, 20:381-387.

4. Chung DC, Maher MM, Faquin WC: Case records of the Massachusetts General Hospital: case 37-2006. A 19-year-old woman with thyroid cancer and lower gastrointestinal bleeding. N Engl J Med 2006, 355:2349-2357.

5. Meyer-Rochow GY, McMullen TP, Gill AJ, Sywak MS, Robinson BG: Intra-abdominal insular thyroid carcinoma metastasis. Thyroid 2009, 19:527-530.

6. Arnous W, Beltran S, Berger N, Bournaud C, Lifante JC, Peix JL, Giammarile F, Sassolas G, Borson-Chazot F: Hürthle cell thyroid carcinoma metastatic to the sigmoid colon. Thyroid 2007, 17:169-173.

7. Song S, Yenice KM, Kopec M, Liauw SL: Image-guided radiotherapy using surgical clips as fiducial markers after prostatectomy: a report of total setup error, required PTV expansion, and dosimetric implications. Radiother Oncol 2012, 103:270-274.

8. Engels B, Soete G, Verellen D, Storme G: Conformal arc radiotherapy for prostate cancer: increased biochemical failure in patients with distended rectum on the planning computed tomogram despite image guidance by implanted markers. Int J Radiat Oncol Biol Phys 2009, 74:388-391.

9. Abbas H, Chang $\mathrm{B}$, Chen ZJ: Motion management in gastrointestinal cancers. J Gastrointest Oncol 2014, 5:223-235.
10. Kirkbride P, Bezjak A, Pater J, Zee B, Palmer MJ, Wong R, Cross P, Gulavita S, Blood P, Sun A, Dundas G, Ganguly PK, Lim J, Chowdhury AD, Kumar SE, Dar AR: Dexamethasone for the prophylaxis of radiation-induced emesis: a National Cancer Institute of Canada Clinical Trials Group phase III study. J Clin Oncol 2000, 18:1960-1966.

11. Dennis K, Nguyen J, Presutti R, DeAngelis C, Tsao M, Danjoux C, Barnes E, Sahgal A, Holden L, Jon F, Wong S, Chow E: Prophylaxis of radiotherapyinduced nausea and vomiting in the palliative treatment of bone metastases. Support Care Cancer 2012, 20:1673-1678.

12. Feyer PC, Maranzano E, Molassiotis A, Roila F, Clark-Snow RA, Jordan K: Radiotherapy-induced nausea and vomiting (RINV): MASCC/ESMO guideline for antiemetics in radiotherapy: update 2009. Support Care Cancer 2011, 19(Suppl 1):S5-S14.

13. Baglan KL, Frazier RC, Yan D, Huang RR, Martinez AA, Robertson JM: The dose-volume relationship of acute small bowel toxicity from concurrent 5-FU-based chemotherapy and radiation therapy for rectal cancer. Int J Radiat Oncol Biol Phys 2002, 52:176-183.

14. Robertson JM, Lockman D, Yan D, Wallace M: The dose-volume relationship of small bowel irradiation and acute grade 3 diarrhea during chemoradiotherapy for rectal cancer. Int J Radiat Oncol Biol Phys 2008, 70:413-418.

15. Kavanagh BD, Pan CC, Dawson LA, Das SK, Li XA, Ten Haken RK, Miften M: Radiation dose-volume effects in the stomach and small bowel. Int J Radiat Oncol Biol Phys 2010, 76(3 Suppl):S101-S107.

16. Bissonnette J-P, Franks KN, Purdie TG, Moseley DJ, Sonke J-J, Jaffray DA, Dawson LA, Bezjak A: Quantifying interfraction and intrafraction tumor motion in lung stereotactic body radiotherapy using respiration-correlated cone beam computed tomography. Int I Radiat Oncol Biol Phys 2009, 75:688-695

17. Nakamura M, Shibuya K, Shiinoki T, Matsuo Y, Nakamura A, Nakata M, Sawada A, Mizowaki T, Hiraoka M: Positional reproducibility of pancreatic tumors under end-exhalation breath-hold conditions using a visual feedback technique. Int J Radiat Oncol Biol Phys 2011, 79:1565-1571.

18. Rosewall T, Chung P, Bayley A, Lockwood G, Alasti H, Bristow R, Kong V, Milosevic M, Catton C: A randomized comparison of interfraction and intrafraction prostate motion with and without abdominal compression. Radiother Oncol 2008, 88:88-94.

19. Rash D, Hagar Y, Cui J, Hunt J-P, Valicenti R, Mayadev J: Interfraction motion of the vaginal apex during postoperative intensity modulated radiation therapy: are we missing the target? Int I Gynecol Cancer 2013, 23:385-392.

20. Case RB, Moseley DJ, Sonke JJ, Eccles CL, Dinniwell RE, Kim J, Bezjak A, Milosevic M, Brock KK, Dawson LA: Interfraction and intrafraction changes in amplitude of breathing motion in stereotactic liver radiotherapy. Int J Radiat Oncol Biol Phys 2010, 77:918-925.

21. Della Biancia C, Yorke E, Kollmeier MA: Image guided radiation therapy for bladder cancer: assessment of bladder motion using implanted fiducial markers. Pract Radiat Oncol 2014, 4:108-115.

22. Van der Horst A, Wognum S, Dávila Fajardo R, de Jong R, van Hooft JE, Fockens $P$, van Tienhoven G, Bel A: Interfractional position variation of pancreatic tumors quantified using intratumoral fiducial markers and daily cone beam computed tomography. Int J Radiat Oncol Biol Phys 2013, 87:202-208

23. Deegan $T$, Owen R, Holt T, Fielding A, Biggs J, Parfitt M, Coates A, Roberts L: Assessment of cone beam $\mathrm{CT}$ registration for prostate radiation therapy: Fiducial marker and soft tissue methods. J Med Imaging Radiat Oncol 2014. http://www.ncbi.nlm.nih.gov/pubmed/24953244. 